\title{
Directed attention and the recognition of pictures
}

\author{
CLAUDIA J. STANNY and GEORGE E. WEAVER \\ Florida State University, Tallahassee, Florida 32306
}

\begin{abstract}
In previous research, Weaver and Stanny (1978) found that immediate recognition memory for one of several pictorial stimuli was facilitated when the target for a forced-choice recognition test was preceded by an attentional cue. In the present experiment, improved recognition for cued stimuli was obtained for stimuli in several serial positions and over filled retention intervals of several minutes. However, no support was obtained for a hypothesis that allocation of additional effort to one stimulus requires termination of processing for other stimuli in the study sequence. Specifically, on trials in which a cue was presented, recognition of targets presented prior to the cue was no poorer than that for targets in analogous study sequences in the absence of a cue.
\end{abstract}

Common to a number of recent accounts of the human memory system is the hypothesis that memory characteristics for a stimulus event are a direct consequence of the type of processing that an observer applies to the stimulus information (e.g., Craik \& Lockhart, 1972; Morris, Bransford, \& Franks, 1977). Recent studies of memory for pictorial information have provided results consistent with such a notion. For example, Frost (1972) and Tversky (1973) have shown that retention of visual stimuli is influenced by the type of retention test the subject expects (recognition or recall), presumably due to changes in the particular stimulus features selected and processed. Similarly, Bower and Karlin (1974) reported that orientation to certain properties of pictures of human faces can affect level of recognition memory for these stimuli.

In a somewhat different context, Weaver and Stanny (1978) have shown that immediate recognition for one of a series of highly similar pictures can be facilitated by indicating to the subjects the particular stimulus that will serve as the target. Thus, in a probe recognition test in which only one of several study stimuli was to be the target, performance was superior when the target was preceded by a cue (in this case, a buzzer). However, the heterogeneous nature of the stimuli (photographs of outdoor scenes) did not provide an opportunity for the direct manipulation of processing strategy, as has been possible in studies of word recall (e.g., Hyde \& Jenkins, 1973) or in studies including homogeneous visual stimuli (e.g., Bower \& Karlin, 1974). Instead, subjects were told only to make what-

This study was conducted by the first author in partial fulfillment of the requirements for the master's degree. The authors wish to acknowledge the helpful criticism of Darryl Bruce during the conduct of this research and on an earlier version of this paper. Requests for reprints should be sent to Claudia J. Stanny, Department of Psychology, Florida State University, Tallahassee, Florida 32306. ever use they could of the information provided by the occurrence of the cue. The finding of superior performance for targets following a cue indicates that subjects can control the allocation of processing effort.

A major goal of the present experiment was the identification of the general parameters of the attentional effect previously reported by the present authors. This may be viewed as a first step in formulating hypotheses about the specific changes in processing that subjects may adopt in order to improve recognition performance for pictorial stimuli. Several changes were made in the cuing procedures introduced by Weaver and Stanny (1978) in an attempt to assess the generality of the effect of an attentional cue on recognition performance. In addition, conditions were introduced that were expected to provide evidence regarding the effect allocation of effort to one stimulus in a series would have on processing of and memory for other stimuli in the study sets.

In the initial demonstration of the cuing effect (Weaver \& Stanny, 1978, Experiment 4), the retention test immediately followed completion of each of a series of five pictures. Thus, it was not possible to determine whether the effect of the cue was merely a transitory maintenance of certain features of the target or involved a more long-term change in the storage of stimulus information. In the present study, a delay was imposed between the sets of study pictures and subsequent recognition tests. In addition, the cue was presented prior to one of two different serial positions in the study sets, whereas only one location was preceded by a cue in the previous research.

Since pilot data indicated that the cue effect would be evident both over longer retention intervals and for several different serial positions, the design of the present experiment also included another manipulation, which was expected to provide insight into the nature of the processing changes that result from the occur- 
rence of an attentional cue. Specifically, the question asked was whether allocating additional processing to one picture in a series of pictures requires the termination of processing of information from other pictures in the series.

If the positive effect of the attentional cue does involve termination of processing for stimuli that precede the cue, recognition for these stimuli should be inferior to what it would have been had no cue been introduced into the study sequence. Such a negative effect has been obtained in studies of directed forgetting for segments of lists of words (Bruce \& Papay, 1970; Epstein, 1972). Alternatively, it is possible that the allocation of additional processing to a pictorial stimulus does not have an effect on the processing of other stimuli in the study sets. If this is the case, items preceding a cue should be recognized as well as are homologous items in trials without the presence of a cue.

\section{METHOD}

\section{Design and Materials}

The stimuli consisted of $35-\mathrm{mm}$ color slides of outdoor scenes. From a large initial stimulus pool, study sets of five similar pictures were formed, all of the pictures in each study set coming from a single content subgrouping (e.g., beach scenes varying only in wave patterns, successive views of a manyhued sunset, etc.). For each study set, one picture was randomly identified as the target stimulus. A test pair was then formed for each study set, composed of a duplicate of the target stimulus and a distractor stimulus from the same content grouping. A total of 28 such sets and corresponding test pairs were formed, four study-test sequences serving as practice trials, the remaining 24 sequences providing data upon which all analyses were based. These were presented in four blocks of six study sequences. As only one picture in each study set served as the target, the procedure constitutes a probe test of recognition memory.

During the presentation of half the study sequences, a cue was inserted during the interstimulus interval (ISI) separating two study stimuli. In contrast to the procedure employed by Weaver and Stanny (1978), in which a buzzer served as the cue, in the present study the cue consisted of the visual presentation of a homogeneous pale blue color field on the screen. The duration of the cue was $1.5 \mathrm{sec}$, following the offset of the previous stimulus and preceding the onset of the subsequent stimulus by approximately $.8 \mathrm{sec}$. The overall brightness of the cue was comparable to that of the study stimuli.

A within-subjects design was employed, consisting of the factorial combination of three variables: cuing condition (cue present or absent), target location (precue or postcue), and cue location (defined by the serial position of the stimulus following the cue, 3 or 5). The last two variables were pseudoclassifications on trials not including a cue. Thus, there were eight types of study-test sequences, four in which a cue was presented before either Serial Position 3 or 5 and in which the target was prior to or subsequent to the cue. For the remaining four study sequences, no cue was presented, but the target was in Serial Position 2 or 3 (corresponding to sequences including a cue before the stimulus in Serial Position 3) or in Serial Position 4 or 5 (corresponding to sequences including a cue before the stimulus in Serial Position 5).

A total of eight counterbalanced presentation arrangements were formed to ensure that each target stimulus appeared equally often in each of the treatment sequences. With a total of 24 study-set sequences, three observations were obtained from each subject under each of the eight types of treatment condi- tion. The order of presentation of study sets and the respective test pairs was held constant for all subjects.

\section{Apparatus and Procedure}

Stimuli were presented on a $1.5 \times 3 \mathrm{~m}$ screen via three Carousel slide projectors controlled by electronic timers. Subjects were tested in small groups of two to six individuals and were seated approximately $1.5 \mathrm{~m}$ from the screen. A small lamp provided low-level ambient illumination throughout the experiment. Following instructions, four practice trials were given, followed by the 24 experimental study sequences grouped in four blocks of 6 study sets each. The test pairs for a block of study sets were presented only after all six study sets were completed

The five stimuli in each study set were presented sequentially in the middle of the screen. Study stimuli (and the homogeneous blue slide) subtended approximately a 40-deg horizontal visual angle. Stimulus duration was $1.5 \mathrm{sec}$, with an ISI of $3 \mathrm{sec}$. The ISI was dark on study sequences, except on those occasions when a cue was introduced. Following the final picture in each study set, a 3-sec interval was provided, during which the experimenter said "ready" and began the presentation of stimuli for the second study set. This procedure continued until all six study sets were completed. The experimenter then warned the subjects that the test pairs were to be presented. The stimuli composing each test pair were presented simultaneously side by side on the screen. Each of the test stimuli subtended approximately $30 \mathrm{deg}$ of horizontal visual angle.

Subjects recorded their choice of the left or right picture as "old" and also indicated the level of confidence in their choice on a 3-point scale. The test was unpaced. Test pairs for each block were presented in the same serial order as the study set stimuli, and target items were randomly assigned to the left or right position, with the constraint that each position occur equally often across the 24 test pairs.

Subjects were informed that cued stimuli would occasionally not be the actual target of the subsequent test pair. However, they were advised that the use of the cue as a study aid would increase the probability of a correct response. To ensure that all five items were studied regardless of cuing conditions, the subjects were also told that there would be a second recognition test at the end of the experiment, in which any item (whether following a cue or not) from any study set could be a test-pair target. Contrary to these instructions, no final test was given.

\section{Subjects}

A total of 72 male and female students at Florida State University served as subjects in partial fulfillment of a course requirement. In order to equalize the number of subjects in each presentation arrangement, additional subjects were deleted by a lottery procedure.

\section{RESULTS AND DISCUSSION}

A confidence score was derived for each response that reflected both the correctness of choice and the level of confidence indicated. These scores could range from 6 (a correct response with highest certainty) to 1 (an incorrect response, but with highest certainty the choice was correct). The means of these scores are presented in Table 1. Although all statistical analyses were computed on the confidence values described above, the same pattern of results is apparent for the percent correct choices (hits), which are also given in Table 1.

When a cue was presented prior to Serial Position 3, recognition of targets in the precue position was not consistently different from that for targets in the same serial position in the absence of a cue. Recognition 
Table 1

Mean Confidence Scores (C) as a Function of Experimental Treatment Condition

\begin{tabular}{ccccc}
\hline & \multicolumn{4}{c}{ Target Location } \\
\cline { 2 - 5 } Cuing & \multicolumn{2}{c}{ Precue } & \multicolumn{2}{c}{ Postcue } \\
\cline { 2 - 5 } Condition & $\mathrm{C}$ & Hits & C & Hits \\
\hline \multirow{4}{*}{ Cue Preceding Serial Position 3 } \\
Present & 4.40 & 74 & 4.98 & 83 \\
Absent & 4.45 & 71 & 4.50 & 73 \\
& \multicolumn{4}{c}{ Cue Preceding Serial Position 5 } \\
Present & 4.56 & 75 & 5.00 & 85 \\
Absent & 4.25 & 69 & 4.40 & 75 \\
\hline
\end{tabular}

Note-Hits = percent correct responses, without regard to confidence level. In cue-absent conditions, target location (preor postcue) and cue location (3 or 5) are pseudoclassifications.

memory for postcue targets, however, was superior to that for stimuli in the same serial position but on trials without a cue. The same pattern of results was observed when the cue was presented prior to Serial Position 5. Thus, regardless of serial position in the study set, recognition performance was superior for postcue targets on trials with a cue. Furthermore, there was no evidence that the change in processing that results in superior recognition for cued targets requires a termination of processing for stimuli that immediately precede the cue.

Anaiysis of variance confirmed the above observations. The main effect of cuing condition (cue present or absent) was statistically reliable $[\mathrm{F}(1,71)=20.04$, $\mathrm{MSe}=2.41]$, as was the main effect of target location (precue or postcue) $[\mathrm{F}(1,71)=17.36, \mathrm{MSe}=2.20]$. The level of significance for all tests in this report was set at the .01 level. Consistent with the observation that the effect of the cue on recognition performance is apparent only for postcue stimuli was the reliable interaction of cuing condition and location of the target $[\mathrm{F}(1,71)=7.84, \mathrm{MSe}=2.53]$. Calculation of the simple effects of cuing condition for targets in the precue and postcue locations, respectively, indicated that there was a statistically reliable difference in confidence scores for targets following the cue $[\mathrm{F}(1,71)=25.08$, $\mathrm{MSe}=2.53]$. However, there was no reliable difference in performance for targets in the precue location associated with the presence or absence of the cue $[F(1,71)=$ $1.39, \mathrm{MSe}=2.53]$. No other main effects or interactions achieved statistical significance in this analysis. Thus, the magnitude of the cue effect was essentially the same for targets in Serial Positions 3 and 5, and there were no consistent differences in performance as a function of serial position on trials without cues.

The most important aspect of these data is the demonstration that directed attention can result in increased recognition for a pictorial stimulus under a wide range of conditions. Thus, it was shown that the effect on recognition persists over relatively long retention intervals, is obtained regardless of serial position of the target in the study sets, and may be obtained with a visual cue (a blue light) as well as with an auditory stimulus (Weaver \& Stanny, 1978). It would thus appear that the phenomenon involves alterations in processing that subjects can control under a variety of situations.

In addition, the finding that recognition of targets in the precue positions was neither superior to nor inferior to that of analogous targets on trials without cues indicates that additional attention can be given to one stimulus in a study set without an effect on the processing of other study stimuli. In general, it appears that subjects are able to allocate a certain, relatively constant amount of processing effort to each item as it is presented (thus, the absence of differences in recognition as a function of serial position on trials in which no cue occurs). However, additional effort can be applied in the processing of a selected individual stimulus. Similar results have been reported recently by Graefe and Watkins (1980), using a poststimulus cuing procedure.

One possible interpretation of these findings is that subjects adopt a strategy for the processing of cued stimuli that involves attention to specific details of the target stimulus. Although the selection of highly similar pictorial stimuli appears to be successful in discouraging the use of simple verbal labels on trials in which no cue is present (cf. Weaver \& Stanny, 1978, p. 61), such a "naming" strategy might be effective for retaining information about a specific stimulus in the study set. If such a label-rehearsal process were employed only on pictures preceded by a cue, it might be expected that there would be little or no effect of cue occurrence on the processing of other stimuli in the study set.

\section{REFERENCES}

Bower, G. H., \& Karlin, M. B. Depth of processing pictures of faces and recognition memory. Journal of Experimental Psychology, 1974, 103, 751-757.

Bruce, D., \& PAPAY, J. P. Primacy effects in single-trial free recall. Journal of Verbal Learning and Verbal Behavior, 1970, 9, 473-486.

Craik, F. I. M., \& Lockhart, R. S. Levels of processing: A framework for memory research. Journal of Verbal Learning and Verbal Behavior, 1972, 11, 671-684.

EPSTEIN, W. Mechanisms of directed forgetting. In G. H. Bower (Ed.), The psychology of learning and motivation: Advances in research and theory (Vol. 6). New York: Academic Press, 1972.

Frost, N. Encoding and retrieval in visual memory tasks. Journal of Experimental Psychology, 1972, 95, 317-326.

Graefe, T. M., \& Watkins, M. J. Picture rehearsal: An effect of selectively attending to pictures no longer in view. Journal of Experimental Psychology: Human Learning and Memory, 1980, 6, 156-162.

HydE, T. S., \& Jenkins, J. J. Recall for words as a function of semantic, graphic, and syntactic orienting tasks. Journal of Verbal Learning and Verbal Behavior, 1973, 12, 471-480.

Morris, C. D., Bransford, J. D., \& Franks, J. J. Levels of processing versus transfer appropriate processing. Journal of Verbal Learning and Verbal Behavior, 1977, 16, 519-533.

TVERSKY, B. Encoding processes in recognition and recall. Cognitive Psychology, 1973, 5, 275-287.

Weaver, G. E., \& StanNY, C. J. Short-term retention of pictorial stimuli as assessed by a probe recognition technique. Journal of Experimental Psychology: Humun Learning and Memory, 1978, 4, 55-65.

(Received for publication April 23, 1980.) 